\title{
Estudio Predictivo del Rendimiento Matemático en PISA 2012: Enfoque de Aprendizaje Frente a la Atribución del Fracaso
}

\author{
Predictive Study of Mathematical Performance in PISA 2012: Focus of Learning \\ Facing the Attribution of Failure
}

\author{
Esperanza Bausela Herreras ${ }^{1}$
}

\begin{abstract}
Resumen
Las matemáticas son críticas para el desarrollo del individuo, siendo varias las variables que pueden predecir un bajo rendimiento en esta competencia. El objetivo del estudio es analizar y predecir el rendimiento matemático en función de atribuciones al fracaso y el enfoque de aprendizaje. Se ha optado por un diseño descriptivo basado en la aplicación de un cuestionario. La asociación entre atribuciones y enfoque de aprendizaje con rendimiento en matemáticas es muy baja pudiendo considerar la independencia de dichas variables y una relación muy débil. La asociación más alta con la competencia matemática, se establece con: "Mi profesor no explicó bien los conceptos esta semana" $[\chi 2=.103 ; p<.001]$ y "Partes importantes vs. Conocimientos previos vs. Aprender de memoria" $[\chi 2=.155 ; p<.001]$. Los estudios de predicción indican que las atribuciones tienen mayor capacidad predictiva del rendimiento matemático. Se recomienda la importancia de trabajar el estilo atribucional para optimizar el rendimiento de todos los estudiantes.
\end{abstract}

Palabras clave: estudios PISA, rendimiento, competencia, atribuciones, enfoque de aprendizaje

\begin{abstract}
Mathematics is critical in the development of the individual. There are several variables that can predict student's future poor performance in the competence of mathematics. The aim of this study was to analyse and predict student's future mathematical performance according to: student's attributions of failure and student's approach to learning. In this study, we used a descriptive research design using survey research based on the application of a questionnaire. The association between student attributions of failure and student's approach to learning, with a focus on performance in mathematics, is very poor considering the independence of these variables and that they are negligibly related. Results in this study, the highest association with mathematical competence is established with: "This week, my teacher did not explain the concepts well enough for me to properly understand them" $[\chi 2=.103 ; p<.001]$ and "Essential aspects vs. Prior knowledge vs. Learning from memory" $[\chi 2=.155 ; p<.001]$. Studies on predicting student's performance indicate that student's attributions have a greater predictive capacity for mathematical performance. The importance of operating with the attributional style in optimising student performance in the whole class is recommended.
\end{abstract}

Keywords: PISA studies, performance, competence, attributions, focus of learning

\footnotetext{
${ }^{1}$ Departamento de Ciencias de la Salud, Universidad Pública de Navarra, España. Correo: esperanza.bausela@unavarra.es 


\section{Introducción}

Son diversas la variables que se asocian con el rendimiento académico como constatan diferentes estudios (Barca, Peralbo, \& Brenlla, 2004; Barca, Almeida, Porto, Peralbo, \& Brenlla, 2012; Bausela, 2016; Bausela, 2018: Gibb, Zhu, Alloy, \& Abramson, 2002; Houston, 2016; Ramudo et al., 2017). Estas variables pueden agruparse bajo tres grandes dimensiones del Modelo "3P" de Biggs (1994): presagio, proceso, producto.

En el presente estudio - desde un enfoque cognitivo - nos centramos en los factores relacionados con el alumno (presagio), concretamente en dos constructos: atribuciones causales, enfoque de aprendizaje y su relación con el rendimiento.

\section{Atribuciones causales y rendimiento}

Una variable que incide en el rendimiento académico es la atribución que el estudiante realiza de su rendimiento siendo objeto de estudio y análisis por diferentes investigadores (Gibb et al., 2002; Liu, Cheng, Chen, \& Wu, 2009).

Weiner (1990, 2010) sintetiza las causas principales para atribuir el éxito o el fracaso en los procesos de aprendizaje a la capacidad, al esfuerzo, a la tarea y a la suerte, apoyándose en tres dimensiones: (i) Lugar de causalidad: internalidad-externalidad; (ii) Estabilidad: estabilidad-inestabilidad; (iii) Controlabilidad: controlabilidad-incontrolabilidad.

Recientes investigaciones (González, 2005; Pintrich \& Schunk, 2006) hacen referencia a la implicación de diferentes variables que pueden incidir en la atribución: profesor, clima del aula, interés del estudiante...siendo las atribuciones causales de mayor complejidad y amplitud que lo inicialmente propuesto por Weiner.

Almaguer (1998) y Woolfolk (1995) estiman que cuando el éxito o el fracaso se atribuyen a factores internos se asocia de forma diferente con el rendimiento académico. Las evidencias apuntan a diferencias en función del nivel de rendimiento, así, los estudiantes con calificaciones más altas poseen un locus de control interno (Almaguer, 1998). Valle, González, Rodríguez, Piñeiro y Suárez (1999), por su parte, comparan las atribuciones causales entre estudiantes universitarios de alto y bajo rendimiento. Los resultados indican que el grupo con alto rendimiento académico atribuye su éxito a su capacidad y esfuerzo, además, de tener un alto concepto de sí mismos. El grupo de bajo rendimiento académico atribuye su éxito al contexto. Estos alumnos reconocen la falta de capacidad y esfuerzo a su bajo rendimiento, mientras que, en otras ocasiones, atribuyen su éxito y fracaso a la suerte.

Según el estudio desarrollado por Choy, O'Grady y Rotgans (2012) las atribuciones son un buen predictor del rendimiento académico. Así, se evidencia que los patrones atribucionales influyen en el desarrollo académico de los adolescentes (Schunk, 1982).

La relación entre estilo de atribución y rendimiento académico se constata en diversos estudios (Gibb, Zhu, Alloy, \& Abramson, 2002; Houston, 2016) como ya hemos comentado en líneas precedentes y se puede explicar desde el concepto de autorregulación. Las habilidades de autorregulación, a su vez, están mediadas por la situación y no son estables (ver modelo propuesto por Zimmerman, 2000).

No obstante, no existe consenso sobre la aplicación de esta teoría en el ámbito escolar, en cuanto al poder predictivo de las atribuciones y sus dimensiones hacia los resultados académicos, por lo menos, no tal y como propone el modelo de Weiner (Navas, Castejón, \& Sampascual, 2000).

Desde el punto de vista empírico los resultados de las asociaciones entre atribuciones y rendimiento muestran, además, algunos resultados contradictorios. Así, Liu et al. (2009) en un estudio longitudinal obtienen que las atribuciones de esfuerzo predicen mayores aumentos en el rendimiento con el tiempo, en comparación con las atribuciones de habilidad. En contraste, otros investigadores aportan evidencias de que las atribuciones de habilidad predicen el logro mejor que las atribuciones de esfuerzo (Meyer, Weir, McClure, Walkey, \& McKenzie, 2009). Watkins y Gutiérrez (2001) constatan que las atribuciones de habilidad y esfuerzo son igualmente predictivos del logro.

Bong (2004), por su parte, perfiló esta asociación, sugiriendo que las atribuciones de capacidad son predictores de logros específicos del contexto pero no de logros en general. Esta 
afirmación es coherente con la teoría de Weiner (1985) y los hallazgos de Liu et al. (2009).

En esta misma dirección apuntan los resultados obtenidos por Tsujimoto et al. (2018) en un estudio comparativo en relación a las atribuciones que hacen a competencia lectora niños con dificultades de aprendizaje de la lectura y desarrollo neurotípico. Los resultados sugieren que las atribuciones de capacidad para la competencia lectora están relacionadas con la capacidad de lectura y/o el déficit, y no con alteraciones generales del lenguaje.

Woodcock \& Vialle (2016) analizan las atribuciones que realizan no sólo los estudiantes, sino también las que realizan los profesores sobre el rendimiento de los estudiantes con dificultades en el proceso de aprendizaje teniendo un impacto en las atribuciones, motivaciones y autoeficacia de los propios estudiantes.

Diversos instrumentos pueden ser aplicados para evaluar las atribuciones en el contexto educativo. Citizenship Attributional Style Questionnaire ha sido recientemente validado (Yazıcıa \& Güvenba, 2017), obteniendo evidencias de sus propiedades psicométricas mucho más amplias que muchos de los instrumentos actualmente utilizados. Estos mismos autores realizan una revisión de los diversos instrumentos que permiten evaluar el estilo atribucional de los estudiantes: (i) The Attributional Style Questionnaire (ASQ; Peterson et al., 1982); (ii) The Cognitive Style Questionnaire (CSQ; Haeffel et al., 2008); (iii) The Occupational Attributional Style Questionnaire (OASQ; Furnham, Sadka, \& Brewin, 1992); (iv) The Academic Attributional Style Questionnaire (AASQ; Peterson, \& Barett, 1987); (v) The Children's Attributional StyleQuestionnaire (CASQ; Seligman et al., 1984); (vi) Escala SIACEPA (Sistema Integrado de Evaluación de Atribuciones Causales y Procesos de Aprendizaje) (Barca, Peralbo, \& Brenlla, 2004).

Para prevenir situaciones negativas $\mathrm{y}$ fomentar las atribuciones adaptativas se creó la técnica AR (Attributional Retraining) que ha sido definida como una intervención cognitiva motivacional por varios autores (Hamm, Perry, Clifton, Chipperfield, \& Boese, 2014). La técnica AR tiene como objetivo modificar el razonamiento explicativo disfuncional de los estudiantes al alentar atribuciones adaptativas de bajo rendimiento (explicaciones internas, controlables e inestables, como falta de esfuerzo y mala estrategia), en lugar de esquemas atribucionales desadaptativos basados en causas estables e incontrolables (como la falta de inteligencia) (Boese et al., 2013). Los hallazgos obtenidos por Matteucci (2017) confirman la efectividad la técnica AR en la reestructuración de explicaciones atributivas estables autodestructivas y sugiere que los objetivos de logro están implicados en la adopción de dimensiones causales adaptativas.

\section{Enfoque de aprendizaje y rendimiento}

Junto con las atribuciones, también las investigaciones señalan que la manera de enfocar las actividades incide en el rendimiento académico (Escanero, Soria, Escanero, \& Guerra, 2013; Freigberg \& Fernández, 2013; González, del Rincon Igea, \& del Rincón Igea, 2011; Ortiz \& Canto, 2013; Ossa \& Lagos, 2013; Wilkinson, Boohan, \& Stevenson, 2013; Yip, 2013).

Inicialmente, estimamos necesario incidir en clarificar la diferencia entre enfoque de aprendizaje y estilo cognitivo, que son utilizados, en ocasiones, como sinónimos, aunque en realidad son dos constructos diferentes, aunque interrelacionados (López \& López, 2013). Los enfoques de aprendizaje son considerados una forma de estilo de aprendizaje (Riding \& Rayner, 1995). El estilo de aprendizaje se entiende como una expresión del estilo cognitivo y la personalidad (Corominas et al., 2006; Ibrahimoglu, Unaldi, Samancioglu y Baglibel, 2013). Este constructo es definido como un conjunto de atributos cognitivos, afectivos y fisiológicos que determinan el modo en que las personas perciben, interaccionan y responden a su ambiente de aprendizaje (Freiberg \& Fernández, 2013)

Biggs (1988) define los enfoques de aprendizaje como los procesos cognitivos que posibilitan a los alumnos percibir y procesar la información proveniente de las diferentes actividades académicas. Se pueden inferir diferentes formas de aprender. Marton y Säljö (1976) y Barca et al. (1999), apoyándose en Biggs diferencian en sus estudios tres tipos de 
acercamiento al aprendizaje: (i) Superficial la información se asimila de forma textual y con un aprendizaje de tipo memorístico (asociacionismo) $y$ se centran en detalles seleccionados y reproducidos con precisión. Las estrategias predominantes son: repetición / memorísticas / mnemotecnia; (ii) Profundo, se trata de desarrollar un aprendizaje significativo, que permita relacionar las nuevas ideas con los conocimientos previos (aprendizaje significativo de Ausubel), maximizando la comprensión. Las estrategias predominantes son: selección, organización o elaboración. Ambos enfoques (superficial y profundo) interrelacionan con componentes motivacionales y estratégicos (motivos y estrategias) (Barca et al., 1999); (iii) De logro que busca sobresalir, destacar y optimizar la organización del tiempo y el esfuerzo con "destrezas de estudio".

Rosario (1999) propone que estos tres enfoques se pueden reducir a dos tipos de enfoques: (i) Enfoque hacia la superficialidad/reproducción y (ii) enfoque de aprendizaje dirigido hacia el significado/comprensión. A estos mismos resultados llega Biggs, Kember y Leung (2001) quienes identifican, igualmente, dos factores que identifican: (i) factor de orientación profunda "deep" y (ii) factor de orientación superficial "surface".

Barca y Peralbo (2002) también consideran que el enfoque de logro debe integrarse dentro del superficial y profundo, dando origen al enfoque de orientación a la superficialidad, (EOR-SP) relacionado con la motivación extrínseca y el enfoque de orientación al significado, (EOR-SG) vinculado a una motivación intrínseca.

En la práctica, no resulta fácil poder identificarlos, así Ruíz y Molina (2011), evidencian en una investigación con universitarios que tienden hacia una combinación de enfoque de logro y enfoque superficial. Freiberg, Berenguer, Fernández y Ledesma (2016), por su parte, encuentran diferencias en los enfoques en función del nivel educativo de los estudiantes universitarios. Así, los estudiantes de primer ciclo tienden a utilizar con mayor predominancia el enfoque superficial que sus compañeros de cursos superiores.

Los enfoques de aprendizaje pueden evaluarse con un cuestionario adaptado por Freiberg y Fernández (2016) y, sobre todo, con el Cuestionario de Procesos de Estudio Revisado RSPQ-2F (Biggs, Kember, \& Leung, 2001), ampliamente utilizado en el ámbito de investigación que evalúa el enfoque: superficial, profundo y de logro (Choy, O'Grady, \& Rotgans, 2012; López, 2009) o con "Situated Goal", Questionnaire for University Students (SGQ-U) (Alonso Tapia et al., 2018).

Study Process Questionnaire (SPQ) (Biggs, 1987) es una escala que evalúa los tres enfoques de aprendizaje, configurado por motivos y estrategias: (i) Enfoque superficial: son estudiantes que presentan una motivación extrínseca, cuya estrategia es centrarse en los esencial para luego reproducirlo. (ii) Enfoque profundo: son estudiantes que están intrínsecamente motivados, cuyo objetivo es comprender y relacionar los contenidos de la tarea. (iii) Enfoque de logro: son estudiantes que lo que les motiva es demostrar su competencia ante los demás. Ha sido aplicado en diversos estudios (Freiberg \& Fernández, 2016; Mohd et al., 2013).

La intervención en relación a los enfoques de aprendizaje viene condicionada entre otras variables por la metodología docente, así, si el docente opta por una metodología expositiva es probable que el estudiante opte por un enfoque superficial o de logro, si por el contrario, el docente opta por metodologías activas (enfoque constructivista) es más probable que el estudiante opte por un enfoque profundo (Gargallo, Suárez, García, Pérez, \& Sahuqillo, 2012).

\section{El presente estudio}

En este contexto nos planteamos analizar y predecir el riesgo de tener bajo, medio y alto rendimiento en la competencia matemática considerando como variables predictoras: atribuciones del fracaso y enfoque de aprendizaje.

Estimamos que las atribuciones que hace el estudiante de sus éxitos y de sus fracasos tienen una mayor capacidad predictiva del rendimiento que el propio enfoque utilice durante el proceso de enseñanza-aprendizaje. 


\section{Método}

No experimental, descriptivo basado en la aplicación de un cuestionario.

\section{Participantes}

En el caso de España (objeto del presente estudio) la muestra está constituida por 25313 jóvenes de 15 años.

La técnica de muestreo utilizada es bietápico por conglomerados. Primero se seleccionan los centros educativos (mínimo de 150 por país) y luego los estudiantes (35 alumnos por centro educativo).

En competencia matemática en PISA 2015 $(\mathrm{M}=495.43$; $\mathrm{DT}=85.79)$, la distribución de los participantes en función de su nivel es: (i) 332 estudiantes presentan alto rendimiento (1.3\%); (ii) 14166 estudiantes presentan rendimiento medio (56\%); (iii) 10815 estudiantes presentan bajo rendimiento $(42.7 \%)$.

\section{Variables de investigación}

Las variables en función del papel que desempeñan en la investigación se clasifican en:

\section{Variable endógena o dependiente:} Competencia matemática. En esta área matemática se han evaluado áreas relacionadas con: los números, el álgebra, la geometría y la estadística interrelacionadas de formas diversas propuestas en el estudio de PISA 2012.

La definición de competencia matemática de PISA 2012 incluye tres aspectos interrelacionados (MEC y OCDE, 2013a): (i) Procesos matemáticos que describen lo que hacen los individuos para relacionar el contexto del problema con las matemáticas y de ese modo resolverlo, y las capacidades que subyacen a esos procesos; (ii) Contenido matemático específico que va a utilizarse en las preguntas de la evaluación; y (iii) Contextos en los que se insertan las preguntas de la evaluación (Ministerio de Educación, Cultura y Deporte, 2013).

Variable exógena o independiente, son dos: Atribuciones causales. Es un cuestionario constituido por seis preguntas con cuatro opciones de respuesta tipo Likert (Muy de acuerdo, De acuerdo, En desacuerdo, Totalmente en desacuerdo) que no dispone de propiedades psicométricas ya que no es una prueba estandarizada. En la Tabla 1 se presentan las preguntas del cuestionario sobre atribuciones al fracaso en matemáticas.

Tabla 1. Preguntas del cuestionario sobre atribuciones del fracaso en matemáticas ${ }^{*}$

\begin{tabular}{|c|c|c|c|c|}
\hline $\begin{array}{l}\text { Atribuciones del } \\
\text { fracaso en las } \\
\text { matemáticas }\end{array}$ & \multicolumn{4}{|c|}{ Respuesta tipo likert } \\
\hline $\begin{array}{l}\text { No soy muy } \\
\text { bueno } \\
\text { resolviendo } \\
\text { problemas } \\
\text { matemáticos. }\end{array}$ & $\begin{array}{c}\text { Muy } \\
\text { probable }\end{array}$ & Probable & $\begin{array}{c}\text { Poco } \\
\text { probable }\end{array}$ & $\begin{array}{c}\text { Nada } \\
\text { probable }\end{array}$ \\
\hline $\begin{array}{l}\text { Mi profesor no } \\
\text { explicó bien los } \\
\text { conceptos esta } \\
\text { semana }\end{array}$ & $\begin{array}{c}\text { Muy } \\
\text { probable }\end{array}$ & Probable & $\begin{array}{c}\text { Poco } \\
\text { probable }\end{array}$ & $\begin{array}{c}\text { Nada } \\
\text { probable }\end{array}$ \\
\hline $\begin{array}{l}\text { Esta semana no } \\
\text { acerté con las } \\
\text { respuestas en el } \\
\text { control. }\end{array}$ & $\begin{array}{c}\text { Muy } \\
\text { probable }\end{array}$ & Probable & $\begin{array}{c}\text { Poco } \\
\text { probable }\end{array}$ & $\begin{array}{c}\text { Nada } \\
\text { probable }\end{array}$ \\
\hline $\begin{array}{l}\text { A veces la } \\
\text { materia del } \\
\text { curso es } \\
\text { demasiado } \\
\text { difícil. }\end{array}$ & $\begin{array}{c}\text { Muy } \\
\text { probable }\end{array}$ & Probable & $\begin{array}{c}\text { Poco } \\
\text { probable }\end{array}$ & $\begin{array}{c}\text { Nada } \\
\text { probable }\end{array}$ \\
\hline $\begin{array}{l}\text { El profesor no } \\
\text { consiguió } \\
\text { interesar a los } \\
\text { alumnos en la } \\
\text { materia. }\end{array}$ & $\begin{array}{c}\text { Muy } \\
\text { probable }\end{array}$ & Probable & $\begin{array}{c}\text { Poco } \\
\text { probable }\end{array}$ & $\begin{array}{c}\text { Nada } \\
\text { probable }\end{array}$ \\
\hline $\begin{array}{l}\text { A veces tengo } \\
\text { mala suerte, } \\
\text { simplemente. }\end{array}$ & $\begin{array}{c}\text { Muy } \\
\text { probable }\end{array}$ & Probable & $\begin{array}{c}\text { Poco } \\
\text { probable }\end{array}$ & $\begin{array}{c}\text { Nada } \\
\text { probable }\end{array}$ \\
\hline $\begin{array}{l}\text { Source: OECD } \\
\text { Assessment } 2012 \\
\text { * Disponible en: } \\
\text { internacionales/pi }\end{array}$ & Program & $\begin{array}{l}\text { le for } \\
\text { mecd.g } \\
\text { 5.html }\end{array}$ & $\begin{array}{l}\text { ernational } \\
\text { s/inee/eva }\end{array}$ & $\begin{array}{l}\text { Studen } \\
\text { aciones }\end{array}$ \\
\hline
\end{tabular}

Enfoque de aprendizaje. El informe español PISA 2012 está centrado en analizar las cuestiones enfocadas a conocer la frecuencia con la que los estudiantes emplean distintas estrategias en su estudio cotidiano. Estas estrategias se clasifican en: (i) Estrategias de control: el alumno controla el proceso de aprendizaje y determina en cada momento lo que necesita saber y aquello que desconoce. (ii) Estrategias de reflexión-relación: el estudiante reflexiona sobre lo que estudia y busca relacionar esos conocimientos con otras asignaturas o con la vida real. (iii) Estrategias memorísticas: el estudiante basa su aprendizaje, sobre todo, en memorizar los conceptos y repetir los ejercicios propuestos en clase.

Es un cuestionario constituido por cuatro preguntas con tres opciones de respuesta a escoger 
una de ellas, la primera respuesta corresponde con estrategias de control, la segunda con estrategias de reflexión-relación y la última de ellas con estrategias memorísticas. Al igual que el anterior cuestionario no dispone de propiedades psicométricas ya que no es una prueba estandarizada. En la Tabla 2 se presentan las preguntas y las respuestas del cuestionario sobre enfoque de aprendizaje en matemáticas.

Tabla 2. Preguntas y respuestas del cuestionario sobre enfoque de aprendizaje en matemáticas ${ }^{*}$

- En cada apartado, escoge entre las tres opciones la que mejor describe cómo enfocas las Matemáticas

- Partes importantes vs. Conocimientos previos vs. Aprender de memoria

- Cuando estudio para un examen de matemáticas, intento determinar qué es lo más importante.

- Cuando estudio para un examen de matemáticas, intento entender nuevos conceptos relacionándolos con cosas que ya sé.

- Cuando estudio para un examen de matemáticas, me aprendo de memoria todo lo que puedo

- Mejorar comprensión vs. Distintos modos de encontrar respuesta vs. Memorizar

- Cuando estudio matemáticas, intento darme cuenta de qué conceptos no he entendido todavía del todo.

- Cuando estudio matemáticas, pienso en distintos modos de hallar la respuesta.

- Cuando estudio matemáticas, repaso para ver si recuero lo que ya he estudiado.

- Otros temas vs. Objetivos de aprendizaje vs Ensayar Problemas

- Cuando estudio matemáticas, empiezo pensando exactamente qué necesito aprender.

- Cuando estudio matemáticas, intento relacionar lo que estudio con cosas que he aprendido en otras asignaturas.

- Cuando estudio matemáticas, repito tan a menudo algunos problemas que siento que los podría resolver dormido.

- Repetir Ejemplos vs. Aplicaciones cotidianas vs. Más información

- Cuando no entiendo algo en matemáticas, siempre busco información para aclarar el problema.

- Pienso en cómo pueden ser útiles en la vida cotidiana las matemáticas que he aprendido.

- Para recordar el método para resolver un problema de matemáticas, me miro los ejemplos una y otra vez.

Source: OECD Programme for International Student Assessment 2012.

* Disponible en: http://www.mecd.gob.es/inee/evaluacionesinternacionales/pisa/pisa-2015.html

\section{Análisis de datos}

Los datos fueron sometidos a análisis descriptivos multivariados (inferenciales): (i)
Asociación (Chi-Cuadrado) y (ii) estudios de predicción (regresión lineal y regresión logística binaria) (aplicando las mismas técnicas que García, Izquierdo, \& Jiménez, 2000).

\section{Resultados}

\section{Estudio de asociación competencia matemática con atribuciones causales $y$ enfoques de aprendizaje}

\section{Atribuciones causales y competencia matemática}

Los resultados obtenidos por los participantes en relación al enfoque de aprendizaje: (i) El $24.6 \%$ de los participantes está de acuerdo y muy de acuerdo con "No soy bueno con los problemas de matemáticas". (ii) El 31.3\% está de acuerdo "El profesor no explica bien". (iii) El 31.3\% está de acuerdo "Malos cálculos, conjeturas...". (iv) El $32.6 \%$ está de acuerdo "La materia (matemáticas) es demasiado dura". (v) El 24.2\% está de acuerdo "El/la profesor/a no consigue interesar a los estudiantes". (vi) El $22.7 \%$ está de acuerdo con la "Mala suerte" como factor causal de su rendimiento en la competencia matemática (Tabla $3)$.

La asociación entre el tipo de atribuciones y el rendimiento en matemáticas es muy baja pudiendo considerar la independencia de dichas variables y una relación muy débil. La asociación más alta se establece entre "Mi profesor no explicó bien los conceptos esta semana" y la competencia matemática $\left[\chi^{2}=.103 ; p<.001\right]$.

\section{Enfoque de aprendizaje $y$ competencia matemática}

Los resultados obtenidos por la muestra participante indican que: (i) El $26.9 \%$ considera que "Cuando estudio para un examen de matemáticas, intento determinar qué es lo más importante"; (ii) El 30.8\% afirma "Cuando estudio matemáticas, intento darme cuenta de qué conceptos no he entendido todavía del todo"; (iii) El $34.8 \%$ señala que "Cuando estudio matemáticas, intento relacionar lo que estudio con cosas que he aprendido en otras asignaturas"; (iv) El 39.2\% indica que "Cuando 
Tabla 3. Atribuciones del fracaso en matemáticas (frecuencia y porcentaje)

\begin{tabular}{|c|c|c|}
\hline Variables & Frecuencia & Porcentaje \\
\hline \multicolumn{3}{|c|}{ No soy muy bueno resolviendo problemas matemáticos. } \\
\hline Muy probable & 2155 & 8.5 \\
\hline Probable & 6224 & 24.6 \\
\hline Poco probable & 6229 & 24.6 \\
\hline Nada probable & 1976 & 7.8 \\
\hline No sabe, no contesta, perdido, inválido & 8729 & 34.5 \\
\hline \multicolumn{3}{|c|}{ Mi profesor no explicó bien los conceptos esta semana } \\
\hline Muy probable & 2699 & 10.7 \\
\hline Probable & 7915 & 31.3 \\
\hline Poco probable & 4640 & 18.3 \\
\hline Nada probable & 1292 & 5.1 \\
\hline No sabe, no contesta, perdido, inválido & 8767 & 34.6 \\
\hline \multicolumn{3}{|c|}{ Esta semana no acerté con las respuestas en el control. } \\
\hline Muy probable & 2699 & 10.7 \\
\hline Probable & 7915 & 31.3 \\
\hline Poco probable & 4640 & 18.3 \\
\hline Nada probable & 1292 & 5.1 \\
\hline No sabe, no contesta, perdido, inválido & 8767 & 34.6 \\
\hline \multicolumn{3}{|c|}{ A veces la materia del curso es demasiado difícil. } \\
\hline Muy probable & 4400 & 17.4 \\
\hline Probable & 8248 & 32.6 \\
\hline Poco probable & 3089 & 12.2 \\
\hline Nada probable & 817 & 3.2 \\
\hline No sabe, no contesta, perdido, inválido & 8759 & 34.6 \\
\hline \multicolumn{3}{|c|}{ El profesor no consiguió interesar a los alumnos en la materia. } \\
\hline Muy probable & 3326 & 13.1 \\
\hline Probable & 6133 & 24.2 \\
\hline Poco probable & 5381 & 21.3 \\
\hline Nada probable & 1724 & 6.8 \\
\hline No sabe, no contesta, perdido, inválido & 8749 & 34.6 \\
\hline \multicolumn{3}{|l|}{ A veces tengo mala suerte, simplemente. } \\
\hline Muy probable & 2286 & 9 \\
\hline Probable & 5740 & 22.7 \\
\hline Poco probable & 5482 & 21.7 \\
\hline Nada probable & 2969 & 11.7 \\
\hline No sabe, no contesta, perdido, inválido & 8836 & 34.9 \\
\hline
\end{tabular}

Source: OECD Programme for International Student Assessment 2012.

no entiendo algo en matemáticas, siempre busco información para aclarar el problema".

En la Tabla 4 se presentan los descriptivos (frecuencia y porcentaje) de la muestra española participante en el uso de enfoque de aprendizaje en matemáticas.

La asociación entre el enfoque de aprendizaje y el rendimiento en matemáticas es muy baja pudiendo considerar la independencia de dichas variables y una relación muy débil. La asociación más alta se establece entre "Partes importantes vs. Conocimientos previos vs. Aprender de memoria" con la competencia matemática $\left[\chi^{2}=.155 ; p<.001\right]$.

\section{Estudio de predicción del rendimiento matemático: atribuciones fracaso y enfoque de aprendizaje}

\section{Regresión lineal múltiple}

La técnica más adecuada para responder al objetivo de la investigación y que se ajusta a la naturaleza de las variables dependiente (continua) es el modelo de regresión lineal múltiples.

El coeficiente de correlación simple es de .269 (el obtenido en el análisis de las correlaciones bivariadas) y su cuadrado .072. Morales Vallejo (2012, p.14) afirma que la correlación múltiple ajustada (R ajustada) es "una estimación de $R$ en la población, no en la muestra" y expresa que el ajuste no es importante con muestras mayores a 100 y menos de 5 predictores. Se encontró un coeficiente de correlación R ajustado igual .0.71.

El modelo global (ANOVA) fue estadísticamente significativo ( $\mathrm{F}=62.137 ; p=.000)$, es decir, el modelo permite suponer la existencia de un efecto real de las variables predictoras sobre la variable dependiente. El modelo se muestra claramente válido para representar los datos. El valor de significación obtenido $(p=.000)$ indica que la probabilidad de que el conjunto de variables 
Tabla 4. Enfoque de aprendizaje en matemáticas (frecuencia y porcentaje)

\begin{tabular}{|c|c|c|}
\hline Variables & Frecuencia & Porcentaje \\
\hline \multicolumn{3}{|l|}{ Partes importantes vs. Conocimientos previos vs. Aprender de memoria } \\
\hline $\begin{array}{l}\text { Cuando estudio para un examen de matemáticas, intento determinar qué es lo más } \\
\text { importante. }\end{array}$ & 6816 & 26.9 \\
\hline $\begin{array}{l}\text { Cuando estudio para un examen de matemáticas, intento entender nuevos } \\
\text { conceptos relacionándolos con cosas que ya sé. }\end{array}$ & 6743 & 26.6 \\
\hline $\begin{array}{l}\text { Cuando estudio para un examen de matemáticas, me aprendo de memoria todo lo } \\
\text { que puedo }\end{array}$ & 2960 & 11.7 \\
\hline No sabe, no contesta, perdido, no válido & 8794 & 34.7 \\
\hline \multicolumn{3}{|l|}{ Mejorar comprensión vs. Distintos modos de encontrar respuesta vs. Memorizar } \\
\hline $\begin{array}{l}\text { Cuando estudio matemáticas, intento darme cuenta de qué conceptos no he } \\
\text { entendido todavía del todo. }\end{array}$ & 7786 & 30.8 \\
\hline Cuando estudio matemáticas, pienso en distintos modos de hallar la respuesta. & 3434 & 13.6 \\
\hline Cuando estudio matemáticas, repaso para ver si recuero lo que ya he estudiado. & 5282 & 20.9 \\
\hline No sabe, no contesta, perdido, no válido & 8811 & 34.8 \\
\hline \multicolumn{3}{|l|}{ Otros temas vs. Objetivos de aprendizaje vs Ensayar Problemas } \\
\hline $\begin{array}{l}\text { Cuando estudio matemáticas, empiezo pensando exactamente qué necesito } \\
\text { aprender. }\end{array}$ & 3122 & 12.3 \\
\hline $\begin{array}{l}\text { Cuando estudio matemáticas, intento relacionar lo que estudio con cosas que he } \\
\text { aprendido en otras asignaturas. }\end{array}$ & 8815 & 34.8 \\
\hline $\begin{array}{l}\text { Cuando estudio matemáticas, repito tan a menudo algunos problemas que siento } \\
\text { que los podría resolver dormido. }\end{array}$ & 4500 & 17.8 \\
\hline No sabe, no contesta, perdido, no válido & 8876 & 35.1 \\
\hline \multicolumn{3}{|l|}{ Repetir Ejemplos vs. Aplicaciones cotidianas vs. Más información } \\
\hline $\begin{array}{l}\text { Cuando no entiendo algo en matemáticas, siempre busco información para aclarar } \\
\text { el problema. }\end{array}$ & 9930 & 39.2 \\
\hline $\begin{array}{l}\text { Pienso en cómo pueden ser útiles en la vida cotidiana las matemáticas que he } \\
\text { aprendido. }\end{array}$ & 2638 & 10.4 \\
\hline $\begin{array}{l}\text { Para recordar el método para resolver un problema de matemáticas, me miro los } \\
\text { ejemplos una y otra vez. }\end{array}$ & 3870 & 15.3 \\
\hline No sabe, no contesta, perdido, inválido & 8875 & 35.1 \\
\hline
\end{tabular}

Source: This study has been development from PISA 2012 (Spanish sample) (elaboración propia).

predictoras introducidas no sea suficiente para aportar explicación de los valores predichos de $\mathrm{Y}$ es nula. Es decir, las variaciones en la variable competencia Matemática se explican significativamente por el conjunto de efectos predictivos identificados.

La Tabla 5 se presenta información sobre la ecuación de regresión y sus coeficientes.

Según la información proporcionada por los coeficientes no estandarizados, la ecuación que representa la regresión de las atribuciones al fracaso y los enfoques de aprendizaje sobre el rendimiento en matemáticas es:

$$
\mathrm{Y}=490.870+12.953 \mathrm{X}_{1}-11.138 \mathrm{X}_{2}-3.966
$$
$\mathrm{X}_{3}+15.259 \mathrm{X}_{4}-4.025 \mathrm{X}_{5}+5.971 \mathrm{X}_{6}-5.738 \mathrm{X}_{7^{-}}$ $7.625 \mathrm{X}_{8}+1.608 \mathrm{X}_{9}+1.028 \mathrm{X}_{10}$

Las variables que tienen mayor peso en la ecuación de predicción del rendimiento en matemáticas están relacionadas con la atribución al fracaso, siendo los coeficientes $\beta$ estandarizados: "No soy тиy bueno resolviendo problemas matemáticos" (.126) y "Mi profesor no explicó bien los conceptos esta semana" (-.115).

Todas las variables incluidas en la ecuación de predicción tienen un peso estadísticamente significativo, a excepción, de dos variables relacionadas con los enfoques de aprendizaje: "Otros temas vs. Objetivos de aprendizaje vs Ensayar Problemas" y "Repetir Ejemplos vs. Aplicaciones cotidianas vs. Más información".

Las puntuaciones $t$ indican que las variables tenidas en cuenta aportan significativamente al modelo de predicción o que los valores obtenidos se pueden generalizar a la población. En este estudio, las variables independientes: (i) "No soy muy bueno resolviendo problemas matemáticos" $(t=10.858 ; p=.000)$. (ii) "Mi profesor no explicó bien los conceptos esta semana" ( $t=-9.302$; $p=.000$ ). (iii) "Esta semana no acerté con las respuestas en el control" ( $t=-3.354 ; p=.001)$. (iv) "A veces la materia del curso es demasiado difícil" ( $t=12.181 ; p=.000)$. (v) "El profesor no consiguió interesar a los alumnos en la materia" $(t=-3.540 ; p=.000)$. (vi) “A veces tengo mala suerte, simplemente" $(t=5.989 ; p=.000)$. (vii) "Partes importantes vs. Conocimientos previos vs. Aprender de memoria" ( $t=-4.651 ; p=.000)$. (viii) "Mejorar comprensión vs. Distintos modos de 
Tabla 5. Regresión lineal múltiple: Coeficientes estandarizados y no estandarizados

\begin{tabular}{|c|c|c|c|c|c|c|c|}
\hline \multirow{2}{*}{ Variables } & \multicolumn{2}{|c|}{$\begin{array}{l}\text { Coeficientes no } \\
\text { estandarizados }\end{array}$} & \multirow{2}{*}{$\begin{array}{c}\text { Coeficientes } \\
\text { estandarizados } \\
\text { Beta }\end{array}$} & \multirow{2}{*}{$\mathrm{t}$} & \multirow{2}{*}{ Sig. } & \multicolumn{2}{|c|}{$\begin{array}{l}95.0 \% \text { intervalo de } \\
\text { confianza para B }\end{array}$} \\
\hline & B & $\begin{array}{c}\text { Error } \\
\text { estándar }\end{array}$ & & & & $\begin{array}{l}\text { Límite } \\
\text { inferior }\end{array}$ & $\begin{array}{c}\text { Límite } \\
\text { superior }\end{array}$ \\
\hline (Constante) & 490.870 & 6.279 & & 78.177 & .000 & 478.562 & 503.179 \\
\hline $\begin{array}{l}\text { No soy muy bueno resolviendo } \\
\text { problemas matemáticos. }\end{array}$ & 12.953 & 1.193 & .126 & 10.858 & .000 & 10.614 & 15.291 \\
\hline $\begin{array}{l}\text { Mi profesor no explicó bien los } \\
\text { conceptos esta semana }\end{array}$ & -11.138 & 1.197 & -.115 & -9.302 & .000 & -13.485 & -8.791 \\
\hline $\begin{array}{l}\text { Esta semana no acerté con las } \\
\text { respuestas en el control. }\end{array}$ & -3.966 & 1.182 & -.038 & -3.354 & .001 & -6.283 & -1.648 \\
\hline $\begin{array}{l}\text { A veces la materia del curso es } \\
\text { demasiado difícil. }\end{array}$ & 15.259 & 1.253 & .145 & 12.181 & .000 & 12.803 & 17.714 \\
\hline $\begin{array}{l}\text { El profesor no consiguió interesar a los } \\
\text { alumnos en la materia. }\end{array}$ & -4.025 & 1.137 & -.044 & -3.540 & .000 & -6.254 & -1.796 \\
\hline $\begin{array}{l}\text { A veces tengo mala suerte, } \\
\text { simplemente. }\end{array}$ & 5.971 & .997 & .066 & 5.989 & .000 & 4.017 & 7.925 \\
\hline $\begin{array}{l}\text { Partes importantes vs. Conocimientos } \\
\text { previos vs. Aprender de memoria }\end{array}$ & -5.738 & 1.234 & -.051 & -4.651 & .000 & -8.157 & -3.319 \\
\hline $\begin{array}{l}\text { Mejorar comprensión vs. Distintos } \\
\text { modos de encontrar respuesta vs. } \\
\text { Memorizar }\end{array}$ & -7.625 & 1.046 & -.080 & -7.293 & .000 & -9.674 & -5.575 \\
\hline $\begin{array}{l}\text { Otros temas vs. Objetivos de } \\
\text { aprendizaje vs Ensayar Problemas }\end{array}$ & 1.608 & 1.333 & .013 & 1.206 & .228 & -1.005 & 4.222 \\
\hline $\begin{array}{l}\text { Repetir Ejemplos vs. Aplicaciones } \\
\text { cotidianas vs. Más información }\end{array}$ & 1.028 & 1.116 & .010 & .921 & .357 & -1.160 & 3.217 \\
\hline
\end{tabular}

Source: This study has been development from PISA 2012 (Spanish sample) (elaboración propia).

encontrar respuesta vs. Memorizar" ( $t=-7.293$; $p=.000)$ aportan significativamente al modelo predictivo de rendimiento en matemáticas.

La variable que tiene más peso en el modelo es "No soy muy bueno resolviendo problemas matemáticos" ya que es la que presenta mayor coeficiente estandarizado $(\beta=.126 ; \quad p=.000)$, seguida de "Mi profesor no explicó bien los conceptos esta semana" $(\beta=-.115 ; p=.000)$.

\section{Regresión logística binaria}

Para estimar el riesgo de tener un rendimiento (bajo, medio y alto) en función de: atribuciones al fracaso y enfoques de aprendizaje.

\section{Predicción de bajo rendimiento}

Prueba de Hosmer y Lemeshow resultó significativo $[\chi 2(8)=16.678 ; p=.034]$ observemos que el p-valor es inferior a .05, aquí la Hipótesis nula es que el modelo no se ajusta a la realidad.

$R^{2}$ [Cox y Snell=.001; Nagelkerke=.002] son bajos, de forma que no se puede predecir el bajo rendimiento en matemáticas a partir de la atribución causal y el enfoque de aprendizaje.

Para la predicción del bajo rendimiento en matemáticas utilizamos la regresión logística binaria categorizando la variable dependiente en bajo rendimiento $(10851 ; 42.7 \%)$ y no bajo rendimiento $(14498 ; 57.3 \%)$. El modelo permite hacer una estimación correcta del $57.3 \%$ de los casos.

Las variables que resultaron estadísticamente significativas fueron variables vinculadas al enfoque de aprendizaje: (i) "Cuando estudio matemáticas, empiezo pensando exactamente qué necesito aprender" tiene un [ $\beta=-.409 ; p=.044]$, el riesgo a tener bajo rendimiento disminuye un $33.6 \%$. (ii) "Cuando estudio matemáticas, intento relacionar lo que estudio con cosas que he aprendido en otras asignaturas" tiene un $[\beta=$ .454; $p=.024]$, el riesgo a tener bajo rendimiento disminuye un $36.5 \%$. (iii) "Cuando estudio matemáticas, repito tan a menudo algunos problemas que siento que los podría resolver dormido" tiene un $[\beta=-.462 ; p=.023]$, el riesgo a tener bajo rendimiento disminuye un $37 \%$. (iv) "No sabe, no contesta, perdido, inválido" tiene un $[\beta=-.421 ; p=.025]$, el riesgo a tener bajo rendimiento disminuye un $34.3 \%$.

\section{Predicción de rendimiento medio}

Prueba de Hosmer y Lemeshow resultó significativo $[\chi 2(8)=10.822 ; p=.212]$ observemos 
que el p-valor es superior a .05, aquí la Hipótesis nula indica que el modelo se ajusta a la realidad.

$R^{2}$ [Cox y Snell $=.001 ;$ Nagelkerke $=.001$ ] son bajos de forma que no se puede predecir el rendimiento medio en matemáticas a partir de la atribución causal y el enfoque de aprendizaje.

Para la predicción del medio rendimiento en matemáticas utilizamos la regresión logística binaria categorizando la variable dependiente en rendimiento medio $(14166 ; 56 \%)$ y no rendimiento medio (11147; 44\%). El modelo permite hacer una estimación correcta del $56 \%$ de los casos.

Las variables que resultaron estadísticamente significativas están vinculadas al enfoque de aprendizaje: (i) "Cuando estudio matemáticas, intento relacionar lo que estudio con cosas que he aprendido en otras asignaturas" tiene un $[\beta=.451 ; p=.025]$, el riesgo a tener rendimiento medio se incrementa un $57 \%$. (ii) "Cuando estudio matemáticas, repito tan a menudo algunos problemas que siento que los podría resolver dormido" tiene un $[\beta=.455 ; p=.25]$, el riesgo a tener rendimiento medio se incrementa un $57.7 \%$. (iii) "Para recordar el método para resolver un problema de matemáticas, me miro los ejemplos una y otra vez" tiene un $[\beta=.079 ; p=.041]$, el riesgo a tener rendimiento medio se incrementa un $8.2 \%$. (iv) "No sabe, no contesta, perdido, inválido" tiene un $[\beta=.42 ; p=.024]$, el riesgo a tener rendimiento medio se incrementa un $52.2 \%$.

\section{Predicción de alto rendimiento}

Prueba de Hosmer y Lemeshow resultó significativo $[\chi 2(8)=4.159 ; p=.843]$ observemos que el $p$-valor es superior a .05 , aquí la Hipótesis nula indica que el modelo se ajusta a la realidad.

$R^{2}$ [Cox y Snell=.001; Nagelkerke=.009] son bajos, de forma que no se puede predecir el rendimiento alto en matemáticas a partir de la atribución causal y el enfoque de aprendizaje.

Para la predicción del alto rendimiento en matemáticas utilizamos la regresión logística binaria categorizando la variable dependiente en alto rendimiento $(332 ; 1.3 \%)$ y no alto rendimiento (24981; 98.7\%). El modelo permite hacer una estimación correcta del $98.7 \%$ de los casos.

Las variables que resultaron estadísticamente significativas están vinculadas a las atribuciones causales: (i) "No soy muy bueno resolviendo problemas matemáticos. Muy probable" tiene un $[\beta=.986 . ; p=.014]$, el riesgo a tener alto rendimiento se reduce en un $91.2 \%$. (ii) "No soy muy bueno resolviendo problemas matemáticos. Probable" tiene un $[\beta=.98 ; p=.008]$, el riesgo a tener alto rendimiento se reduce en un $92.4 \%$. (iii) "No soy muy bueno resolviendo problemas matemáticos. Poco probable" tiene un $[\beta=.985$; $p=.010]$, el riesgo a tener alto rendimiento se reduce en un $92 \%$. (iv) "No soy muy bueno resolviendo problemas matemáticos. Nada probable" tiene un $[\beta=1.029 ; p=.006]$, el riesgo a tener alto rendimiento se reduce en un $94 \%$.

\section{Discusión}

Los resultados del presente estudio confirman la hipótesis investigación, que no todas las variables (atribución al fracaso escolar y enfoque de aprendizaje del propio estudiante) tienen la misma capacidad predictiva del riesgo de tener bajo rendimiento en la competencia matemática en PISA 2012, estando en sintonía con los resultados obtenidos en otros estudios (Aeepactamannil, 2014; Aeepactamannil \& Lee, 2014; Areepattannil \& Caleon, 2013; Bong (2004); Meyer, Weir, McClure, Walkey, \& McKenzie, 2009).

Se confirma que las atribuciones causales tienen mayor capacidad predictiva que el enfoque de aprendizaje en la predicción del bajo rendimiento en la competencia matemática. Estos resultados se encuentran en la misma dirección que los resultados obtenidos por Gibb et al. (2002) y Liu, Cheng, Chen y Wu (2009) y con el estudio desarrollado por Choy, O'Grady y Rotgans (2012) quienes consideran que las atribuciones son un buen predictor del rendimiento académico. Son diversos los estudios que han evidenciado diferencias en las atribuciones en función del nivel de rendimiento, lo que justifica que en este estudio se hayan analizado las atribuciones de los participantes en función de su nivel de rendimiento (bajo, medio y alto) - encontrando diferencias en relación al mismo - que se unen a las encontradas en otros estudios (Valle, González, Rodríguez, Piñeiro, \& Suárez, 1999).

Es importante entender estos factores y conocer su influencia para poner más atención a las 
creencias de los estudiantes sobre sus capacidades hacia las matemáticas que condicionara no sólo su rendimiento sino también sus expectativas (Manassero \& Vázquez, 1995), así como entender que no tienen el mismo peso en estudiantes con alto que con bajo rendimiento, como constata Tsujimoto et al. (2018) en el estudio de la ompetencia lectora.

No es posible establecer relaciones de causa efecto entre las variables objeto de estudio (atribuciones y enfoque de aprendizaje) y el rendimiento, pero sí nos proporciona algunos indicaciones sobre buenas prácticas educativas que permitan prevenir el bajo rendimiento en esta competencia, incidiendo, por ejemplo, en el sistema de atribuciones del estudiante considerando su perfil de rendimiento, siendo demostrada y constatada su efectividad por Matteucci (2017) la técnica AR (Attributional Retraining).

Considerando los resultados obtenidos, puede ser recomendable optar por metodologías de enseñanza que faciliten un aprendizaje significativo en detrimento de un aprendizaje memorístico y técnicas de estudio que contribuyan al desarrollo de atribuciones adaptativas tanto el éxito como para el bajo rendimiento por parte del por el propio sujeto, así como diseñar propuestas de intervención psicopedagógica que inician en AR (Attributional Retraining) que ha sido definida como una intervención cognitiva motivacional efectiva por varios autores (Hamm, Perry, Clifton, Chipperfield, \& Boese, 2014).

En este estudio también se ha analizado los enfoques de aprendizaje. Los resultados indican una asociación entre el enfoque por el que opta el estudiante y el rendimiento muy débil, encontrándose en la misma dirección que los obtenidos por otros investigadores (Escanero, Soria, Escanero, \& Guerra, 2013; Freigberg \& Fernández, 2013; González, del Rincon Igea, \& del Rincón Igea, 2011; Ortiz \& Canto, 2013; Ossa \& Lagos, 2013; Wilkinson, Boohan, \& Stevenson, 2013; Yip, 2013).

En nuestro estudio es una variable con menor capacidad predictiva en comparación con las atribuciones causales, no obstante, no puede obviarse su peso e importancia, de ahí, que también puede ser recomendable incidir sobre ella (enfoque de aprendizaje) en el proceso psicoinstruccional (Gargallo, Suárez, García, Pérez, \& Sahuqillo, 2012).

\section{Limitaciones y prospectiva de futuro}

Una de las limitaciones del estudio es no haber analizado la posible interacción de los estilos atribucionales y enfoques de aprendizaje como determinantes del rendimiento académico (Barca, Pestti, Brenlla, \& Santamaría, 2000) en competencia matemática.

Estimamos como futura línea de investigación analizar la atribución al fracaso y el enfoque de aprendizaje como variables predictoras del bajo rendimiento del resto de competencias evaluadas en PISA 2015: Lectura, Ciencias y competencia Financiera. Podemos pensar que la atribución y el enfoque de aprendizaje son variables que pueden incidir de forma general y similar en todas las competencias $\mathrm{o}$, por el contrario, el estudiante puede realizar diferentes atribuciones y optar por diferentes enfoques de aprendizaje en función de la competencia que se está evaluando.

\section{Referencias}

Almaguer, T. (1998). El desarrollo del alumno: Características y estilos de aprendizaje. México: Trillas.

Alonso-Tapia, J., Nieto, C., Merino, E., Huertas, J. A., \& Ruiz, M. (2018) Assessment of learning goals in university students from the perspective of "person-situation interaction": The Situated Goals Questionnaire (SGQ-U). Estudios de Psicología, 39, 20-57. doi:10.1080/02109395.2017.1412707

Areepattamannil, S., \& Caleon, I. S. (2013). Relationships of cognitive and metacognitive learning strategies to mathematics achievement in four high-performing East Asian education systems. The Journal of Genetic Psychology, 174, 696-702. doi:10.1080/00221325.2013.799057

Areepattamannil, S., \& Lee, D. H. L. (2014). Linking immigrant parents' educational expectations and aspirations to their children's school performance. The Journal of Genetic Psychology, 175, 51-57.

doi:10.1080/00221325.2013.799061 
Barca, A., Almeida, A., Porto, A., Peralbo, M., \& Brenlla, J. C. (2012). Motivación escolar y rendimiento: Impacto de metas académicas, de estrategias de aprendizaje y autoeficacia. Anales de Psicología, 28(3), 848-859.

Barca, A., Brenlla, J. C., Santamaría, S., \& González, E. (1999). Estrategias y enfoques de aprendizaje, contextos familiares $y$ rendimiento académico en el alumnado de educación secundaria: indicadores para un análisis causal. Revista Galego-Portuguesa de Psicología e Educación, 3(4), 229-272.

Barca, A., \& Peralbo, M. (2002). Informe Final del Proyecto FEDER/ESOG-Galicia: 1FD970283. Los contextos de aprendizaje $y$ desarrollo en la Educación Secundaria Obligatoria (ESO): Perspectivas de intervención psicoeducativa sobre el Fracaso escolar en la comunidad Autónoma de Galicia. Madrid: Dirección General de Investigación (I+D). Ministerio de Ciencia y Tecnología.

Barca, A., Peralbo, M., \& Brenlla, J. C. (2004). Atribuciones causales $y$ enfoques de aprendizaje: La escala SIACEPA. Psicothema, 16(1), 94-103.

Barca, A., Pestti, R., Brenlla, J. C., \& Santamaría, S. (2000). Enfoques de aprendizaje, estilos atribucionales y rendimiento académico en una muestra de alumnos de educación secundaria de Brasil. Actas del V Congreso Galego - Portugués de Psicopedagogía, 769791.

Barca, A., Porto, A., Vicente, F., Brenlla, J. C., \& Morán, H. (2008). La interacción de estilos atribucionales y enfoques de aprendizaje como determinantes del rendimiento académico. In J. A. González-Pienda y J. C. Núñez. Psicología y educación: un lugar de encuentro. $V$ Congreso Internacional de Psicología y Educación (pp. 670-688). Oviedo: Ediciones de la Universidad de Oviedo.

Bausela, E. (2016). Análisis comparativo de la probabilidad de tener bajo rendimiento en función del sexo en las competencias PISA 2012. Revista Iberoamericana de Diagnóstico y Evaluación - e Avaliação Psicológica, 41, 58-65.
Bausela, E. (2018). PISA 2012: Ansiedad y bajo rendimiento en competencia matemática. Revista Iberoamericana de Diagnóstico y Evaluación - e Avaliação Psicológica, 46, 161-173. doi:10.21865/RIDEP46.1.12

Biggs, J. B. (1987). The Study Processes Questionnaire Manual. Victoria: Australian Council for Educational Research.

Biggs, J. B. (1988). Assessing study approaches to learning. Australian Psychologist, 23, 197206. doi:10.1080/00050068808255604Ç

Biggs, J. B. (1994). Approaches to learning: Nature and measurement of The International Encyclopedia of Education, vol. 1 (2nd ed.), pp. 319-322. Oxford: Pergamon Press

Biggs, J. B., Kember, D., \& Leung, D. (2001). The Revised Two Factor Study Process Questionnaire: R-SPQ-2F. British Journal of Educational Psychology, 71, 133-149. doi:10.1348/000709901158433

Boese, G. D., Stewart, T. L., Perry, R. P., \& Hamm, J. M. (2013). Assisting failure-prone individuals to navigate achievement transitions using a cognitive motivationtreatment (attributional retraining). Journal of Applied Social Psychology, 43, 1946-1955. doi:10.1111/jasp.12139

Bong, M. (2004). Academic motivation in selfefficacy, task value, achievement goal orientations, and attributional beliefs. Journal of Educational Research, 97, 287-297. doi:10.3200/JOER.97.6.287-298

Bull, R., \& Scherif, G. (2001). Executive functioning as a predictor of children's mathematics ability: Inhibition, switching, and working memory. Developmental Neuropsychology, $\quad 19, \quad$ 273-293. doi:10.1207/S15326942DN1903_3

Choy, J. L., O'Grady, G., \& Rotgans, J. I. (2012). Is the Study Process Questionnaire (SPQ) a good predictor of academic achievement? Examining the mediating role of achievementrelated classroom behaviors. Instructional Science, 40, 159-172. doi:10.1007/s11251-011-9171-8

Cirino, P. T., Carlson, C. D., Francis, D. J., \& Fletcher, J. M. (2004). Phonological processing and calculation skill in Spanish speaking English language learners. Paper presented at the International 
Neuropsychological Society 32 nd Annual Meeting. Baltimore, MD.

Compton, D. L., Fuchs, L. S., Fuchs, D., Lambert, W., \& Hamlett, C. (2012). The cognitive and academic profiles of reading and mathematics learning disabilities. Journal of Learning Disabilities, 45, 79-95. doi:10.1177/0022219410393012

Corominas, E., Tesouro, M., \& Teixidó, J. (2006). Vinculación de los enfoques de aprendizaje con los intereses profesionales y los rasgos de personalidad. Aportaciones a la innovación del proceso de enseñanza y aprendizaje en la educación superior. Revista de Investigación Educativa 24 (2), 443-473.

Escanero, J., Soria, S., Escanero, E., \& Guerra, M. (2013). Influencia de los estilos de aprendizaje y la metacognición en el rendimiento académico de los estudiantes de fisiología. Fundación Educación Médica, 16(1), 23-29. doi:10.4321/s2014-98322013000100005

Freiberg, A., \& Fernández, M. (2013). Cuestionario Honey - Alonso de estilos de aprendizaje: Análisis de sus propiedades psicométricas en estudiantes universitarios. Suma Psicológica, 10, 103-117.

Freiberg, A., \& Fernández, M. (2016). Enfoques de aprendizaje según el R-SPQ-2F. Análisis de sus propiedades psicométricas en estudiantes universitarios de Buenos Aires. Revista Colombiana de Psicología, 25(2), 307-329. doi:10.15446/rcp.v25n2.51874

Freiberg, A., Berenguer, D., Fernández, M., \& Ledesma, R. (2016). Estilos, estrategias y enfoques de aprendizaje en estudiantes universitarios de Buenos Aires. Psicodebate, 17(1), 9-34. doi:10.18682/pd.v17i1.626

Furnham, A., Sadka, V., \& Brewin, C. R. (1992). The development of occupational style questionnaire. Journal of Organizational Behavior, 13, 27-39.

García, M.V., Alvarado, J.M., \& Jiménez, A. (2000). La predicción del rendimiento académico: regresión lineal versus regresión logística. Psicothema, 12(2), 248-252.

Gargallo, B. (2000). Procedimientos. Estrategias de aprendizaje. Su naturaleza, enseñanza y evaluación. Valencia: Humanidades Pedagogía.
Gargallo, B., Suárez, J.M., García, E., Pérez, C., \& Sahuqillo, P. (2012). Enfoques de aprendizaje en estudiantes universitarios excelentes y en estudiantes medios. Revista Española de Pedagogía, 252, 185-200.

Geary, D. C., Hamson, C. O., \& Hoard, M. K. (2000). Numerical and arithmetical cognition: A longitudinal study of process and concept deficits in children with learning disability. Journal of Experimental Child Psychology, 77, 236-263. doi:10.1006/jecp.2000.2561

Geary, D. C., Horad, M. K., \& Hamson, C. O. (1999). Numerical and arithmetical cognition: Patterms of functions and deficits in children at risk for mathematical disability. Journal of Experimental Child Psychology, 74, 213-239. doi:10.1006/jecp.1999.2515

Gibb, B.E., Zhu, L., Alloy, L.B., \& Abramson, L. Y. (2002). Attributional styles and academic achievement in university students: A longitudinal investigation. Cognitive Therapy and Research, 26(3), 309-315. doi:10.1023/A:1016072810255

González, A. (2005). Motivación académica: Teoría, aplicación y evaluación. Madrid: Pirámide.

González, J., del Rincón Igea, B., \& del Rincón Igea, D. (2011). Estructura latente $\mathrm{y}$ consistencia interna del R-SPQ-2F: Reinterpretando los enfoques de aprendizaje en el EEES. Revista Investigativa de Educación, 29(2), 277-293.

Haeffel, G. J., Gibb, B. E., Metalsky, G. I., Alloey, L. B., Abramson, L. Y., Hankin, B. L., et al. (2008). Measuring cognitive vulnerability to depres-sion: Development and validation of the cognitive style questionnaire. Clinical Psychological Review, 28, 824-836.

Hamm, J. M., Perry, R. P., Clifton, R. A., Chipperfield, J. G., \& Boese, G. D. (2014). Attributional retraining: A motivation treatment with differential psychoso-cial and performance benefits for failure prone individuals in competitive achievement settings. Basic and Applied Social Psychology, 36(3), 221-237. doi:10.1080/01973533.2014.890623

Houston, D. M. (2016). Revisiting the relationship between attributional style and academic 
performance. Journal of Applied Social Psychology, 46, 192-200. doi:10.1111/jasp.12356

Ibrahimoglu, N., Unaldi, I., Samancioglu, M., \& Baglibel, M. (2013). The relationship between personality traits and learning styles: A cluster analysis. Asian Journal of Management Sciences and Education, 2(3), 93-108.

Kohl, N., Sullivan, A.L., Sadeh, S., \& Zoplouglu, C. (2015). Longitudinal mathematics development of students with learning disabilities and students without disabilities: A comparison of linear, quadratic, and picewise linear mixed effects models. Journal of School Psychology, 53, 105-120. doi:10.1016/j.jsp.2014.12.002

Lander, K., Bevan, A., \& Butterworth, B. (2004). Developmental dyscalculia and basic numerical capacities: A study of 8-9 year old students. Cognition, 92, 99-125. doi:10.1016/j.cognition.2003.11.004

Landerl, K., \& Kölle, C. (2009). Typical and atypical development of basic numerical skills in elementary school. Journal of Experimental Child Psychology, 103, 546-565. doi:10.1016/j.jecp.2008.12.006.

Liu, K. S., Cheng, Y. Y, Chen, Y. L., \& Wu, Y. Y. (2009). Longitudinal effects of educational expectations and achievement attributions on adolescents' academic achievements. Adolescence, 44, 909-924.

López, M. (2009). Estilos de aprendizaje. Relación con motivación y estrategias. Revista Estilos de Aprendizaje 4(4), 1-21.

López, M., \& López, A. I. (2013). Los enfoques de aprendizaje. Revisión conceptual y de investigación. Revista Colombiana de Educación, 64, 131-153.

Manassero, M. A., \& Vázquez, A. (1995). La atribución causal como determinante de las expectativas. Psicothema, 7(2), 361-376.

Marton, F., \& Säljö, R. (1976). On qualitative differences in learning - I: Outcome and process. British Journal of Educational Psychology, 46, 4-11. doi:10.1111/j.2044-8279.1976.tb02980.x

Matteucci, M. C. (2017). Attributional retraining and achievement goals: An exploratory study on theoretical and empirical relationship. Revue Européenne de Psychologie Appliquée 67 (2017) 279-289. doi:10.1016/j.erap.2017.08.004
Meyer, L. H., McClure, J., Walkey, F., Weir, K. F., \& McKenzie, L. (2009). Secondary student motivation orientations and standards-based achievement outcomes. British Journal of Educational Psychology, 79, 273-293. doi:10.1348/000709908X354591

Ministerio de Educación, Cultura y Deporte (2013). Marcos y pruebas de evaluación de PISA 2012: Matemáticas, Lectura y Ciencias [PISA 2012 Assessment and Analytical Framework. Mathematics, Reading, Science, Problem Solving and Financial Literacy]. Madrid: Autor.

Mohd, Z. et al. (2013). Assessing student approaches to learning: A case of business students at the Faculty of Business Management, UiTM. Procedia - Social and Behavioral Sciences 90, 904-913. doi:10.1016/j.sbspro.2013.07.167

Navas, L., Castejón, J.L., \& Sampascual, G. (2000). Un contraste del modelo atribucional de la motivación de Weiner en contextos educativos. Revista de Psicología Social, 15, 69-85.

OECD (2005). PISA 2003. Technical Report, Organisation for Economic Co-operation and Development

[URL:http://www.oecd.org/pisa/data/2015technical-report/.Fecha de consulta: 06/04/2018]

Ortiz, A. F., \& Canto, P. J. (2013). Estilos de aprendizaje y rendimiento académico en estudiantes de Ingeniería en México. Revista Estilos de Aprendizaje, 11(11) [URL:https://www2.uned.es/revistaestilosdea prendizaje/numero_11/articulos/articulo_11.p df. Fecha de consulta: 06/04/2018]

Ossa, C., \& Lagos, N. (2013). Estilos de aprendizaje y rendimiento académico en estudiantes de Pedagogía de Educación General Básica (primaria) de una universidad pública en Chile. Revista Estilos de Aprendizaje, 11(6), 178-189.

Peterson, C., \& Barett, L. (1987). Explanatory style and academic performance among university freshman. Journal of Personality and Social Psychology, 53, 603-607.

Peterson, C., Semmel, A., Baeyer, C. V., Abramson, L. Y., Metalsky, G. I., \& Seligman, M. E. P. (1982). The attributional 
style questionnaire. Cognitive Therapy and Research, 6, 287-300.

Pintrich, P.R., \& Schunk, D.H. (2006). Motivación en contextos educativos. Teoría, investigación y aplicaciones ( $2^{\mathrm{a}}$ ed.). Madrid: Pearson Educación.

Presentación, M. J., Siegenthaler, R., Pinto, V., Mercader, J., \& Miranda, A. (2015). Competencias matemáticas y funcionamiento ejecutivo en preescolar: Evaluación clínica y ecológica. Revista de Neurología, 20(1), 6582.

Ramudo, I., Brenlla, J. C., Barca, A., \& Peralbo, M. (2017). Enfoques de aprendizaje, autoeficacia y rendimiento académico en el alumnado de bachillerato: Implicaciones para la enseñanza. Revista de Estudios $e$ Investigación en Psicología y Educación. Extr.(1), A1-139. doi:10.17979/reipe.2017.0.01.2435

Riding, R., \& Rayner, S. (1995). The information superhighway and individualised learning. Educational Psychology 15, 365-378. doi:10.1080/0144341950150402

Rosario, P. (1999). Variáveis Cognitivomotivacionais na aprendizagem: As "Abordagens ao Estudo" em alunos do Ensino Secundário. Braga: Universidade do Minho.

Ruíz, B., \& Molina, M. (2011). Evaluación de estrategias, motivos y enfoques de aprendizaje para la identificación del perfil de ingreso de estudiantes universitarios. Encuentros, 1, 2134.

Schunk, D. H. (1982). Effects of effort attributional feedback on children's perceived self-efficacy and achievement. Journal of Educational Psychology, 74(4), 548-556.

Seligman, M. E. P., Petorson, C., Kaslow, N. J., Tanenbaum, R. L., Alloy, L. B., \& Abramson, L. Y. (1984). Explanatory style and depressive symptoms among school children. Journal of Abnormal Psychology, 93, 235238.

Shalev, R. S. (2004). Developmental dyscalculia. Journal of Child Neurology, 19, 765-771. doi:10.1177/08830738040190100601

Shalev, R. S., Auerbach, J., Manor, O., \& Gross, V. (2000). Developmental dyscalculia: Prevalence and prognosis. European Child Adolescent Psychiatry, 9 (Suppl 2), II58-II64.
Shell, D. F., \& Husman, J. (2008). Control, motivation, affect, and strategic selfregulation in the college classroom: A multidimensional phenomenon. Journal of Educational Psychology, 100, 443-459. doi:10.1037/0022-0663.100.2.443

Siegel, L. S., \& Ryan, E. B. (1989). The development of working memory in normally achievement and subtypes of learning disabled children. Child Development, 60, 973-980.

Tsujimoto, K. C. et al. (2018). Achievement attributions are associated with specific rather than general learning delays. Learning and Individual Differences 64, 8-21. doi:10.1016/j.lindif.2018.04.002

Valle, A., González, R., Rodríguez, S., Piñeiro, I., \& Suárez, J.M. (1999). Atribuciones causales, autoconcepto y motivación en estudiantes con alto y bajo rendimiento académico. Revista Española de Pedagogía, 214, 525-546.

Watkins, D., \& Gutiérrez, M. (2001). Causal relationships among self-concept, attributions, and achievement in Filipino students. The Journal of Social Psychology, 130, 625-631. doi 10.1080/00224545.1990.9922954

Weiner, B. (2010). Attribution theory. In The Corsini Encyclopedia of Psychology (eds I. B. Weiner and W. E. Craighead). doi:10.1002/9780470479216.corpsy0098

Wilkinson, T., Boohan, M., \& Stevenson, M. (2013). Does learning style influence academic perfomance indifferent forms of assessment. Journal of Anatomy, 224(3), 304308. doi:10.1111/joa.12126

Woodcock, S., \& Vialle, W. (2016). An examination of pre-service teachers' attributions for students with specific learning difficulties. Learning and Individual Differences $\quad 45, \quad 252-259$. doi:10.1016/j.lindif.2015.12.021

Woolfolk, A. (1995). Psicología Educativa. México: Prentice Hall Hispanoamericana, S.A.

Yazıcıa, S., \& Güvenba, S. (2017). Development and validation of a Citizenship Attributional Style Questionnaire. The Social Science Journal 54, 403-410. doi:10.1016/j.soscij.2017.06.002.

Yip, M. (2013). Learning strategies and their relationships to academic performance of high 
school students in Hong Kong. Educational Psychology: An International Journal of Experimental Educational Educational Psychology, 33(7), 817-827. doi:10.1080/01443410.2013.794493

Zimmerman, B. J. (2000). Attaining selfregulation: A social cognitive perspective, In Boekaerts, M., Pintrich, P. R., \& Zeidner, M. (Eds.). Handbook of self-regulation. San Diego, CA: Academic Press.

Zimmerman, B. J., \& Martinez, M. (1986). Development of a structured interview for assessing students use of self-regulated learning strategies. American Educational Research Journal, 23, 614-628. 